\title{
Power Quality Monitoring and Disturbances Classification based on Autoencoder and Neural Network for Electrical Power Supply
}

\author{
A.D. Gonzalez-Abreu ${ }^{1}$, V. Martínez ${ }^{2}$, M. Delgado-Prieto ${ }^{2}$, J.J. Saucedo-Dorantes ${ }^{1}$ and R.A. Osornio- \\ $\operatorname{Rios}^{1}$
}

${ }^{1}$ HSPdigital CA-Mecatronica Engineering Faculty, Autonomous University of Queretaro

San Juan del Rio 76806, Queretaro (Mexico), agonzalez63@alumnos.uaq.mx, raosornio@hspdigital.org,jsaucedo@hspdigital.org

\author{
${ }^{2}$ MCIA Research Center Department of Electronic Engineering, Technical University of Catalonia (UPC) Barcelona, \\ (Spain), \\ victor.martinez@upc.edu, miguel.delgado@mcia.upc.edu
}

\begin{abstract}
The analysis and monitoring of Power Quality (PQ) are a research topic that has concerned with the scientific community in recent years. Several studies have been realized, in which, the object of study regard different approaches; the impact of deficient power supply, the effects caused by the charges in the system or methodologies for the detection, identification and classification of the phenomena that are referred as PQ disturbances, these problematics must be faced to counter the negative effects generated. In this paper is presented two approaches for the characterization and classification of various PQ disturbances, the techniques are involved in the application of subjects related to artificial intelligence; machine learning and deep learning, which in recent years have been used in different areas of study with a good performance for the applications developed. A comparative from these two techniques is performed; the first, machine learning technique, linear discriminant analysis, and the second technique proposed is a deep learning tool called autoencoder. The methodology is tested with a case study with real signals that contain variations in the voltage signal, the results from each technique are presented and the conclusions indicate the comparative realized.
\end{abstract}

Keywords. Power Quality, Machine Learning, Deep Learning, Linear Discriminant Analysis, Autoencoder.

\section{Introduction}

The analysis and monitoring of Power Quality (PQ) represent a trending research topic that has being attracting the scientific community attention since the last decade [1]. In fact, the monitoring of the electrical power supply has become an important aspect in most electrical based application [2], the industrial systems represent an important field of study since the effects of a deficient power supply may impact on undesirable behaviours of the related electrical equipment and machinery. Different studies about (PQ) have been carried out about, the effects that deficient power supply could have on industrial systems. An example of this work is the study presented by L. Morales-Velazquez et al., where the effects caused by different electrical charges in the system is analysed in terms of detection and identification of disturbances. The aim of studies like this is to study the performance of different methodologies to obtain earlier and reliable detection to minimize potential failures and their propagation for electrical equipment protection [3]. The impact that the integration of renewable energy sources on the power system also have been studied, the increasing integration of renewable energy sources has led to new challenges on network planning step and operation [4].

Indeed, a formal definition of $\mathrm{PQ}$ is provided by the IEEE std 1159 [5], referred to as the correct electrical power supply respecting a predefined amplitude and frequency. The norm describes also a set of undesired variations named as PQ disturbances. The standard includes a comprehensive list describing each of these disturbances such as voltage amplitude change, named sag in case of voltage decrease and swell in case of voltage increase.

Thus, the detection and identification of such PQ disturbances are of major interest in recent years. The first step to face this challenge is the estimation of numerical features to characterise waveform. The selection of significant features allows the representation of different disturbances. The consideration of a reduced set of features to reach such capability is important to allow increased performances of the posterior detection and identification algorithms [6]. In this regard, several techniques and methodologies, mainly, focused on transformations as Wavelet based, Fourier or S transform as traditional methods [7]. Nevertheless, others approaches, as the work presented in [8], propose new PQ indexes based on higher order statistic measurements. Thus, it is required often specific work in the feature calculation and feature reduction stages for a proper classification of PQ disturbances, with the aim to avoid problems referred to the loss of information or limits in data variability. 
In this regard, the introduction of artificial intelligence (AI) as new mathematical tools for data characterization and modelling, have resulted in different methodologies applied in a variety of applications, such as images processing, health care, business, robotics or control among others. Indeed, the use of AI based techniques contributes to improve models applied to predict the responses of systems under analysis in which classic methods are limited. In the context of AI, machine learning represents a subarea of AI that involves that the "machine" learns from a specific dataset a certain pattern or behaviour with the aim to predict or recognize patterns in a system. There are multiple techniques in this field, such as Support Vector Machines (SVM), $k$-Nearest Neighbours, Principal Component Analysis, Linear Discriminant Analysis (LDA) Decision Trees, etc. A representative work related with the use of such techniques is the one presented in [9], where a detection and classification of PQ disturbances is faced considering electrical signals considering PCA to reduce the dimension of the acquired features and the application of SVM for posterior classification.

In this regard, deep learning represents a new trend that has been considered recently. Deep learning results from the limitations of classical neural network structures trained by means of backpropagation technique in font of multiple patterns. Unlike machine learning, deep learning deals with more complex models to describe system behaviours, this means that deep learning is supported by high dimensional structures capable to deal with highly nonlinear responses and complex pattern extraction scenarios. In consequence, deep learning models are able to face feature calculation and feature reduction while most machine learning techniques require two stages process. Related works that take advantage of the characteristics that deep learning offers in terms of PQ analysis and monitoring is the work presented in [10], in which a method based on deep convolutional neural networks is used to detect and classify PQ disturbances. The work uses multiple units to extract the features and reduce the overfitting, establishing a comparative with other related works based on traditional methods. Also, in [11], it is presented a singular spectrum analysis and fast discrete curvelet transform, the levels generated from the signals under analysis are used as features and the classification stage involves the use of a deep convolutional neural network and it is compared with SVM classifiers used in others methods existing.

Considering such current works in the field, the aim of this work lies in the proposal of a methodology for monitoring and diagnosis of PQ disturbances based on autoencoder and NN. The methodology faces the detection and identification, and is validated in front of the ideal electrical signal condition and four distinct PQ disturbances, that is, sag, swell, harmonics and fluctuations. The contribution of the work is the analysis of performance from a deep learning technique and the comparison with classic methodologies for PQ monitoring. The benefits and advantages of deep learning techniques have not been discussed widely and this work proposes such analysis in terms of detection and identification performances. The proposed methodology is validated considering a set of synthetic signals and experimental data.

\section{Power Quality disturbances}

As aforementioned, any deviation from the ideal voltage or current can be defined as a PQ disturbance [12]. In this regard, equation (1) represents a pure waveform of the electrical signal:

$$
V(t)=V_{m} \sin (\omega t+\varphi)
$$

where $V(t)$ is the sinusoidal voltage, $V_{m}$ is the amplitude of the signal, $\omega$ is the angular frequency y $\varphi$ is the phase of the signal.

According to the detailed description of the IEEE Std 1159 in regard with the variations of $\mathrm{PQ}$, in the category of short-duration root-mean-square variations, it is included the terms sag and swell. The definitions for these terms define a sag as a decrease in rms voltage which is between $0.1 \mathrm{pu}$ (per unit), and $0.9 \mathrm{pu}$, and swell as the increases in rms voltage above $1.1 \mathrm{pu}$, in both cases the durations range from 0.5 cycles to $1 \mathrm{~min}$. Another defined disturbance is related with sinusoidal voltages or currents having frequencies that are integer multiples of the operation frequency of the supply system, this type of disturbances is referred as harmonics and is included in the category of waveform distortion. Finally, voltage fluctuations are systematic variations of the voltage envelope or a series of random voltage changes, the magnitude of which does not normally exceed the voltage ranges of $0.95 \mathrm{pu}$ to $1.05 \mathrm{pu}$ according to [5].

Complete information about the disturbances and images of these will be presented in the final version of the paper.

\section{Feature Reduction Techniques}

\section{A. Linear Discriminant Analysis}

Classic monitoring strategies consider stages of feature calculation, feature selection and feature reduction. The feature calculation is a stage where a high dimensional set of features is estimated for pattern characterization. Most of the features estimated in this stage present correlated and non-useful information, which minimizes the performance of the overall monitoring methodology. In order to minimize such effects, a feature reduction technique is considered to reduce an original high dimensional feature set into a reduced dimensional space emphasizing non-correlated and significant information. In this regard, the LDA is considered as a useful technique to face multi-class problems. LDA is a supervised technique that aims to find a new projection by maximizing linear separation among the considered classes. The most discriminative and representative information is considered due to LDA attempts to maximize the linear separation between different classes [13].

\section{B. Autoencoder}

The autoencoder technique is a neural network based structure trained to replicate its input at its output. The training of an autoencoder is unsupervised, that is, no 
labeled data is required. The training process is still based on the optimization of a cost function. The cost function measures the error between the input and its resulting reconstruction at the output. The autoencoder structure is composed of an encoder and a decoder, and can have multiple layers. Traditionally, autoencoder was used for dimensionality reduction or feature extraction [14]. Fig. 1 shows the structure of an autoencoder. The basic autoencoder structure consists of three main components, an encoder, a hidden layer and a decoder, the encoder is the input layer, in the hidden size is the representation of the input in a dimension minor to the input layer, the decoder takes the information compressed and return to the original dimension that was in the input layer.

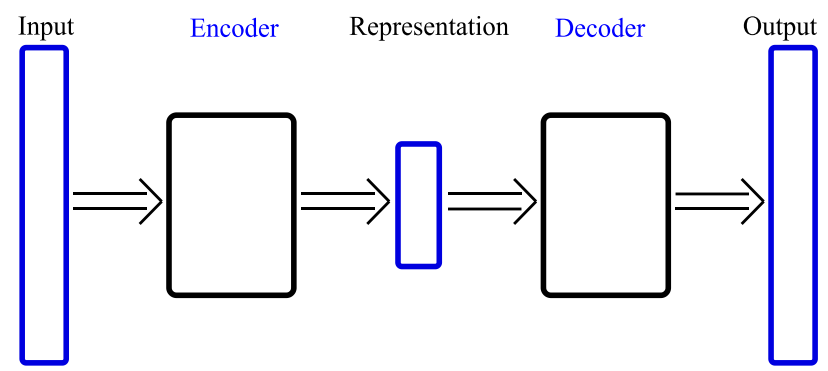

Fig. 1. Representation of an autoencoder structure.

Several autoencoders can be used for the intention to reduce o compress the input data in several steps, passing from a high dimensionality to a low dimensionality without losing the characteristic of features for the input with major relevance to characterizing our data. This scheme is named as stacked autoencoders and consists of the take the encoder part from the autoencoders used to compress the data or called for the intention of this work, feature extraction and feature reduction. A representation of stacked autoencoders is shown in Fig. 2.

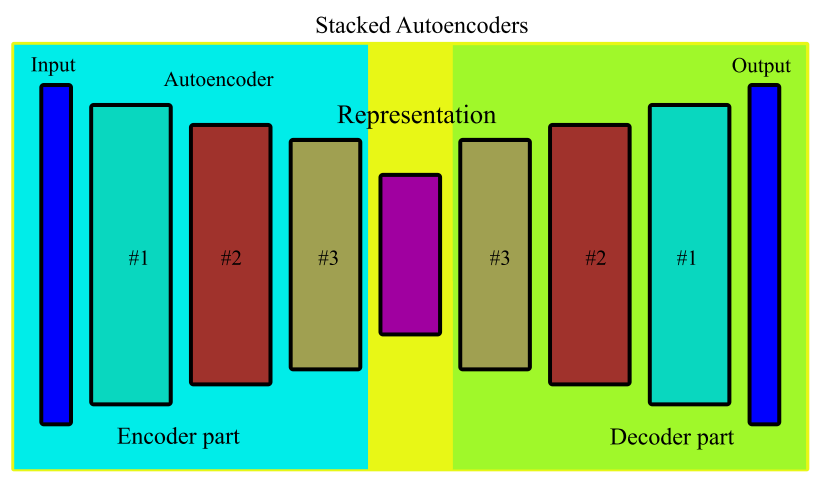

Fig. 2. Three stacked autoencoders.

A discussion about the related works concerning with the use of LDA and autoencoder will be described in this part of the section with the intention to show theoretical aspects and limitations.

\section{Methodology}

The proposed methodology includes feature extraction, feature reduction and classification stages applied to PQ disturbances as shown in the flow chart of Fig. 3. The methodology includes a neural network for final classification. The methodology will be compared with the classical approach based on LDA+NN, that is, considering the use of LDA for feature reduction.

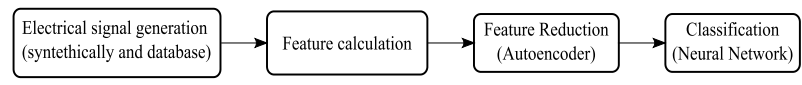

Fig. 3. Flow chart of the proposed methodology using a deep learning approach for extract and reduced features in PQ disturbances detection and classification.

The first stage of the proposed methodology includes the consideration of the electrical signals, including synthetic and experimental ones in this work. An initial set of synthetic signals containing the disturbances under analysis is, first, considered, and then, the evaluation with signals from an experimental database that contains multiple phenomena is evaluated.

Once the representative signals are generated, the next stage in the flow chart correspond to the feature calculation. In this regard, a set of statistical time domainbased is used. The statistical set is shown in Table I.

Table I - Set of statistical time domain-based features

\begin{tabular}{|c|c|c|}
\hline Indicator & Equation & \\
\hline Mean & $\bar{x}=\frac{1}{n} \cdot \sum_{k=1}^{n}\left|x_{k}\right|$ & (2) \\
\hline $\begin{array}{l}\text { Maximum } \\
\text { Value }\end{array}$ & $\hat{x}=\max (x)$ & (3) \\
\hline $\begin{array}{l}\text { Root Mean } \\
\text { Square }\end{array}$ & $R M S=\sqrt{\frac{1}{n}} \cdot \sum_{k=1}^{n}\left(x_{k}\right)^{2}$ & (4) \\
\hline $\begin{array}{c}\text { Square root } \\
\text { mean }\end{array}$ & $S R M=\left(\frac{1}{n} \cdot \sum_{k=1}^{n} \sqrt{\left|x_{k}\right|}\right)^{2}$ & (5) \\
\hline $\begin{array}{l}\text { Deviation } \\
\text { Standard }\end{array}$ & $\sigma=\sqrt{\frac{1}{n} \cdot \sum_{k=1}^{n}\left(x_{k}-\bar{x}\right)^{2}}$ & (6) \\
\hline Variance & $\sigma^{2}=\frac{1}{n} \cdot \sum^{n}\left(x_{k}-\bar{x}\right)^{2}$ & (7) \\
\hline $\begin{array}{l}\text { RMS } \\
\text { Shape } \\
\text { Factor }\end{array}$ & $S F_{R M S}=\frac{R M S}{\frac{1}{n} \cdot \sum_{k=1}^{n}\left|x_{k}\right|}$ & (8) \\
\hline $\begin{array}{l}\text { SRM } \\
\text { Shape } \\
\text { Factor }\end{array}$ & $S F_{S R M}=\frac{S R M}{\frac{1}{n} \cdot \sum_{k=1}^{n}\left|x_{k}\right|}$ & (9) \\
\hline $\begin{array}{l}\text { Crest } \\
\text { Factor }\end{array}$ & $C F=\frac{\bar{x}}{R M S}$ & $(10)$ \\
\hline $\begin{array}{l}\text { Latitude } \\
\text { Factor }\end{array}$ & $L F=\frac{\bar{x}}{S R M}$ & (11) \\
\hline $\begin{array}{l}\text { Impulse } \\
\text { Factor }\end{array}$ & $I F=\frac{\bar{x}}{\frac{1}{n} \cdot \sum_{k=1}^{n}\left|x_{k}\right|}$ & (12) \\
\hline Skewness & $S_{k}=\frac{E\left[\left(x_{k}-\bar{x}\right)^{3}\right]}{\sigma^{3}}$ & (13) \\
\hline Kurtosis & $k_{u}=\frac{E\left[\left(x_{k}-\bar{x}\right)^{4}\right]}{\sigma^{4}}$ & (14) \\
\hline $5^{\circ}$ Moment & $5 t h M=\frac{E\left[\left(x_{k}-\bar{x}\right)^{5}\right]}{\sigma^{5}}$ & (15) \\
\hline $6^{\circ}$ Moment & $6 t h M=\frac{E\left[\left(x_{k}-\bar{x}\right)^{6}\right]}{\sigma^{6}}$ & (16) \\
\hline
\end{tabular}

Once the features are calculated, the feature reduction stage follows. In this stage, from the 15 statistical time 
based-domain features, a new set with the most representative data is obtained. To achieve this, the Fischer score is used, which provides a value or punctuation of the features that are most representative for each class referred to the normal or ideal condition.

With the new set of selected features, the LDA or feature reduction stage follows. In this stage, first, the use of autoencoder and the use of LDA is included. For the case of autoencoder, there are two ways to face the flow chart, in the first one the input of the autoencoder is the signal and, in the second, the input is the set of statistical time domain features. Thus, in the first way, the autoencoder is used as feature extraction, feature selection and feature reduction, and in the second way, the set of statistical indicators the autoencoder only works as feature selection and feature reduction. In this paper, the two considerations are considered and compared.

The number of autoencoders layers is five, following the sizes indicated in Table II.

Table II - Number of neurons in the hidden layer for each autoencoder.

\begin{tabular}{|c|c|}
\hline $\begin{array}{c}\text { No. of } \\
\text { autoencoder }\end{array}$ & $\begin{array}{c}\text { No. of neurons in } \\
\text { the hidden layer }\end{array}$ \\
\hline 1 & 500 \\
\hline 2 & 100 \\
\hline 3 & 50 \\
\hline 4 & 10 \\
\hline 5 & 2 \\
\hline
\end{tabular}

For all the autoencoders the number of epochs established is 500, and the hyperparameters as L2Weight Regularization, Sparsity Proportion and Sparsity Regularization were established in $0.00001,0.01$ and 0.00001 respectively. It is important to mention that only the encoder part of the autoencoders is used. The selection of the values of the hyperparameters is inspired by other works related to the use of autoencoders [15].

The last part of the proposed flow chart is the classification. In this proposed methodology a neural network with SoftMax function is used to perform the classification task, the parameters of the network are: 100 number of epochs and 10 number of neurons in the hidden layer. As aforementioned, the activation function is SoftMax, because is the most used to classification task. In the case of autoencoder, as the autoencoder is a neural network, then to the structure of the encoders stacked only is added one more layer to the end, the classification layer based in SoftMax function.

\section{Experimental Set Up}

As aforementioned the first stage of the methodology is based on the generation of representative electricals signals. The electrical signals are generated using Matlab platform. These signals simulate the five conditions or classes under analysis in this work: the ideal voltage waveform, sag, swell, harmonics and fluctuations. Additionally, to the ideal signals, noise has been added to simulate real conditions. The level of added noise is $50 \mathrm{~dB}$ to each one of the conditions. Fig. 4 show the resulting signals generated to simulate the four PQ disturbances.

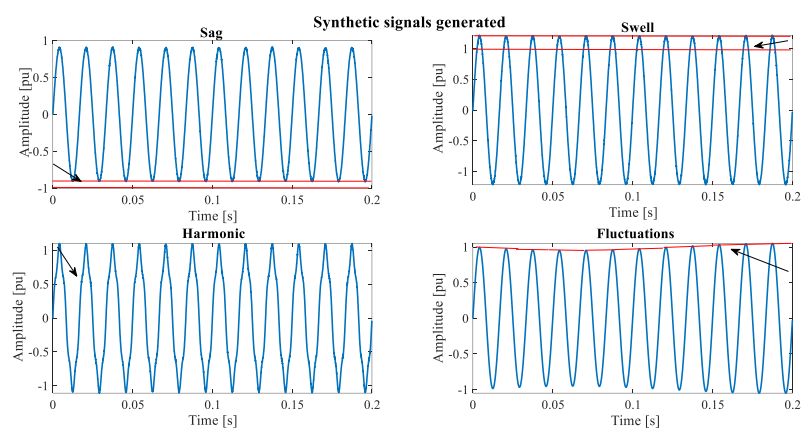

Fig. 4. Electrical signals generated to represent the four PQ disturbances under analysis.

Once generated the signals, the set of statistical time domain-based is calculated. Then, the matrix generated for the calculation of the new statistical set about the synthetic signals is generated computing LDA. The new matrix has then a reduced dimensional space, in which the classes are known and represented in a 2-dimensional space.

Once obtained the representation for the two techniques, a signal obtained from a study case is tested in the two approaches. In the case of machine learning based approach, the procedure is the same, the set of statistical time based-domain is calculated and selected the most representatives and, later, after LDA projection is calculated. The projection of these new signals is superposed over the classification carried out with the synthetic signals, this indicates if the signals contain a PQ disturbance or, otherwise, there are in the normal/ideal condition

This information will be completed in the final version of the paper.

\section{Results and Discussions}

The 2-dimensional projection resulting from the feature reduction stage and the posterior projection of the classification as qualitative analysis. Also, it is pretended to show the accuracy of the classification from a quantitative analysis point of view. The projection of the LDA with the know classes is shown in Fig. 5.

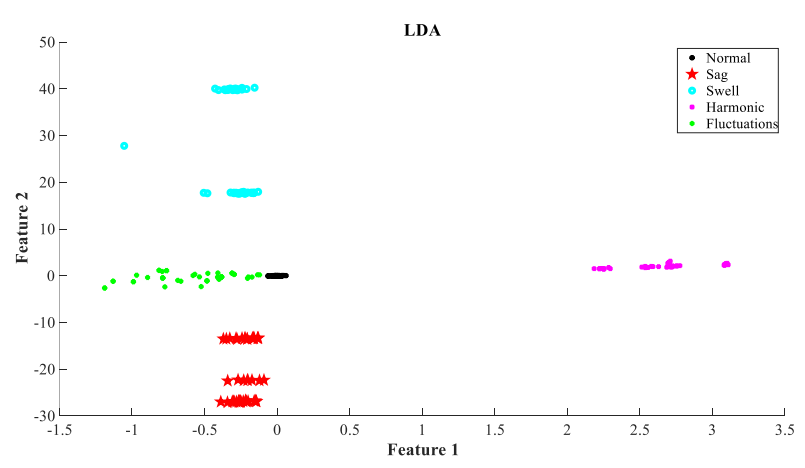

Fig. 5. LDA projection of the synthetic signals. 


\section{Conclusions}

This information will be completed in the final version of the paper.

\section{References}

[1] J. Li, Z. Teng, Q. Tang, and J. Song, "Detection and Classification of Power Quality Disturbances Using Double Resolution S-Transform and DAG-SVMs," IEEE Trans. Instrum. Meas., vol. 65, no. 10, pp. 2302-2312, 2016.

[2] S. M. Blair, C. D. Booth, G. Williamson, A. Poralis, and V. Turnham, "Automatically Detecting and Correcting Errors in Power Quality Monitoring Data," IEEE Trans. Power Deliv., 2017.

[3] L. Morales-Velazquez, R. de J. Romero-Troncoso, G. HerreraRuiz, D. Morinigo-Sotelo, and R. A. Osornio-Rios, "Smart sensor network for power quality monitoring in electrical installations," Meas. J. Int. Meas. Confed., vol. 103, pp. 133$142,2017$.

[4] R. Bayindir, S. Demirbas, E. Irmak, U. Cetinkaya, A. Ova, and M. Yesil, "Effects of renewable energy sources on the power system," Proc. - 2016 IEEE Int. Power Electron. Motion Control Conf. PEMC 2016, pp. 388-393, 2016.

[5] IEEE Standards Coordinating Committee 22 on Power Quality, IEEE Recommended Practice for Monitoring Electric Power Quality, vol. 2019. 1995.

[6] S. Karasu and Z. Saraç, "Investigation of power quality disturbances by using 2D discrete orthonormal S-transform, machine learning and multi-objective evolutionary algorithms," Swarm Evol. Comput., vol. 44, no. November 2018, pp. 1060-1072, 2019.

[7] G. Liu, F. Li, G. Wen, S. Ning, and S. Zheng, "Classification of power quality disturbances based on independent component analysis and support vector machine," Int. Conf. Wavelet Anal. Pattern Recognit., pp. 115-123, 2013.

[8] O. Florencias-Oliveros, A. Agüera-Pérez, J. J. González-de-laRosa, J.-C. Palomares-Salas, and J.-M. Sierra-Fernández, "A novel instrument for power quality monitoring based in higher-order statistics: a dynamic triggering index for the smart grid," Renew. Energy Power Qual. J., vol. 1, no. 15, pp. 43-48, 2017.

[9] P. K. A. Kumar, V. J. Vijayalakshmi, J. Karpagam, and C. K. Hemapriya, "Classification of power quality events using support vector machine and S-Transform," Proc. 2016 2nd Int. Conf. Contemp. Comput. Informatics, IC3I 2016, vol. 7, pp. 279-284, 2016.

[10]S. Wang and H. Chen, "A novel deep learning method for the classification of power quality disturbances using deep convolutional neural network," Appl. Energy, vol. 235, no. November 2018, pp. 1126-1140, 2019.

[11]H. Liu, F. Hussain, Y. Shen, S. Arif, A. Nazir, and M. Abubakar, "Complex power quality disturbances classification via curvelet transform and deep learning," Electr. Power Syst. Res., vol. 163, no. March, pp. 1-9, 2018.

[12]M. H. J. Bollen and I. Y. H. Gu, Signal Processing of Power Quality Disturbances. 2005.

[13]S. Jamali, A. R. Farsa, and N. Ghaffarzadeh, "Identification of optimal features for fast and accurate classification of power quality disturbances," Meas. J. Int. Meas. Confed., vol. 116, no. August 2017, pp. 565-574, 2018.

[14]I. Goodfellow, Y. Bengio, and A. Courville, Deep Learning. MIT Press, 2016.

[15]W. Qiu, Q. Tang, J. Liu, Z. Teng, and W. Yao, "Power Quality Disturbances Recognition Using Modified S Transform and Parallel Stack Sparse Auto-encoder," Electr. Power Syst. Res., vol. 174, no. February, p. 105876, 2019. 